九州大学学術情報リポジトリ

Kyushu University Institutional Repository

\title{
LATTICE PATHS RESTRICTED BY TWO PARALLEL HYPERPLANES
}

Sato, Masako

Department of Mathematical Sciences, College of Engineering, University of Osaka Prefecture

Sado, Taishin

Department of Mathematical Sciences, College of Engineering, University of Osaka Prefecture

https://doi.org/10.5109/13371

出版情報: Bulletin of informatics and cybernetics. 21 (3/4), pp.97-105，1985-03. Research Association of Statistical Sciences

バージョン :

権利関係 : 


\title{
LATTICE PATHS RESTRICTED BY TWO PARALLEL HYPERPLANES
}

By

\section{Masako SATO* and Taishin SADO*}

\begin{abstract}
In the present paper, a lattice path in the nonnegative orthant in the $(k+1)$-dimensional integer lattice is considered. The generating functions are obtained for the numbers of lattice paths restricted by two parallel hyperplanes satisfying various conditions and are expressed by rational functions of $k$-variables. Results include those by Sato and Cong [16].
\end{abstract}

\section{Introduction}

Let us consider paths starting from the origin in the non-negative orthant of the $(k+1)$-dimensional integer lattice space with coordinate variables $Z_{1}, \cdots, Z_{k+1}$. A lattice path (abbreviated by LP throughout) is a path which makes one unit length jump in the positive direction along one of $(k+1)$-axes $Z_{1}, \cdots, Z_{k+1}$ at each step.

A general introduction to the theory of lattice path combinatorics and its applications in various fields are compiled in Mohanty [1].

As is well known, the number of LP's from the origin to a point $\left(n_{1}, \cdots, n_{k}, n_{k+1}\right)$ in the $(k+1)$-dimensional lattice space is given by a multinomial coefficient

$$
\left(\begin{array}{l}
n \\
n
\end{array}\right)=\left(\begin{array}{c}
n \\
n_{1}, \cdots, n_{k}
\end{array}\right)=\frac{n(n-1) \cdots\left(n-\sum_{i=1}^{k} n_{i}+1\right)}{n_{1} ! \cdots n_{k} !}
$$

where $n=\sum_{i=1}^{k+1} n_{i}$ and $\boldsymbol{n}=\left(n_{1}, \cdots, n_{k}\right)$ for $n_{i} \geqq 0, i=1, \cdots, k$.

In what follows, we denote by bold letters $k$-tupples of non-negative integers or variables and define a dot product $\boldsymbol{d} \cdot \boldsymbol{Z}=\sum_{i=1}^{k} d_{i} Z_{i}$ for $\boldsymbol{d}=\left(d_{1}, \cdots, d_{k}\right)$ and $\boldsymbol{Z}=\left(Z_{1}, \cdots, Z_{k}\right)$. And we assume that $d_{1}, \cdots, d_{k}$ are positive integers and $a, b$ and $c$ are non-negative integers.

Among those on the lattice path, much attention has been paid on the problems concerning the number of LP's restricted by given hyperplane(s) in the $(k+1)$-dimensional space. Consider a problem counting the number of LP's starting from the origin and terminating at $(\boldsymbol{n}, \boldsymbol{d} \cdot \boldsymbol{n}+a)$ without crossing a given hyperplane $Z_{k+1}=\boldsymbol{d} \cdot \boldsymbol{Z}$. Mohanty ([2], [4]) showed that the number is given by

* Department of Mathematical Sciences, College of Engineering, University of Osaka Prefecture, Sakai, Osaka 591, Japan. 


$$
A_{\boldsymbol{n}}(a+1, \boldsymbol{d}+\mathbf{1})=\frac{a+1}{a+1+(\boldsymbol{d}+\mathbf{1}) \cdot \boldsymbol{n}}\left(\begin{array}{c}
a+1+(\boldsymbol{d}+\mathbf{1}) \cdot \boldsymbol{n} \\
\boldsymbol{n}
\end{array}\right),
$$

where $1=(1,1, \cdots, 1)$. He derived a convolution identity on the expressions (1.2) and also the one relating multinomial coefficients with (1.2). His results were generalizations of Gould's results for $k=1([5]-[8])$.

Note that $A_{n}(1,2)$ (a case that $k=1, d_{1}=1$, and $a=1$ ) is the so-called $n$-th Catalan number which plays an important role in combinatorial problems ([9]-[13]). See also Speed [14] for a geometric and probabilistic interpretation of (1.2).

Now consider two hyperplanes $P_{1}: Z_{k+1}=\boldsymbol{d} \cdot \boldsymbol{Z}-b$ and $P_{2}: Z_{k+1}=\boldsymbol{d} \cdot \boldsymbol{Z}+c$. Denote by $W_{\boldsymbol{d}}(\boldsymbol{n}, a, b)$ and $T_{\boldsymbol{d}}(\boldsymbol{n}, a, b, c)(0 \leqq a \leqq b+c)$ the number of LP's from the origin to $(\boldsymbol{n}, \boldsymbol{d} \cdot \boldsymbol{n}+a-b)$ without crossing the hyperplane $P_{1}$ and that of LP's from the origin to the same point $(\boldsymbol{n}, \boldsymbol{d} \cdot \boldsymbol{n}+a-b)$ which cross neither $P_{1}$ nor $P_{2}$, respectively. Obtain the expressions $W_{\boldsymbol{d}}(\boldsymbol{n}, a, b)$ and $T_{\boldsymbol{d}}(\boldsymbol{n}, a, b, c)$.

For a case that $k=1$, this problem has been already solved by Sato and Cong([15], [16]).

In the present paper, we shall extend their results to a multidimensional case. In section 2 , we shall give an expression for $W_{\boldsymbol{d}}(\boldsymbol{n}, a, b)$ from which we derive its generating function. In section 3 , we give a generating function for $T_{\boldsymbol{d}}(\boldsymbol{n}, a, b, c)$ by making use of the results in section 2 . One will see that both generating functions are expressed in terms of polynomials

$$
\begin{aligned}
\varphi_{\boldsymbol{d}}(\boldsymbol{X}, n) & =\sum_{0 \leq d \cdot \boldsymbol{l} \leq n}\left(\begin{array}{c}
(\boldsymbol{d}+\mathbf{1}) \cdot \boldsymbol{l}-n-1 \\
\boldsymbol{l}
\end{array}\right) \boldsymbol{X}^{\boldsymbol{l}} \\
& =\sum_{0 \leq d \cdot \boldsymbol{l} \leq n}\left(\begin{array}{c}
n-\boldsymbol{d} \cdot \boldsymbol{l} \\
\boldsymbol{l}
\end{array}\right)(-\boldsymbol{X})^{\boldsymbol{l}}, \quad n \geqq 0,
\end{aligned}
$$

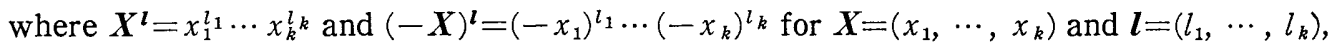
and where $\varphi_{1}(\boldsymbol{X}, n)$ are closely related to the Chebyshev polynomials of the second kind. In section 4 , we consider a special case that $\boldsymbol{d}=\mathbf{1}$. By expanding the generating function for $T_{1}(n, a, b, c)$ obtained in section 3 , we give an explicit expression for $T_{1}(\boldsymbol{n}, a, b, c)$.

\section{Generating Function of $\left\{W_{\boldsymbol{d}}(\boldsymbol{n}, a, b), \boldsymbol{n} \geqq \mathbf{0}\right\}$}

Let us introduce a generating function

$$
W_{\boldsymbol{d}}(\boldsymbol{X}, a, b)=\sum_{n \geq 0} W_{\boldsymbol{d}}(\boldsymbol{n}, a, b) \boldsymbol{X}^{n},
$$

where $W_{\boldsymbol{d}}(\mathbf{0}, a, b)=1$. If $a<b$ and $0 \leqq \boldsymbol{d} \cdot \boldsymbol{n}<b-a, W_{\boldsymbol{d}}(\boldsymbol{n}, a, b)$ can not be evaluated since the terminal point $(\boldsymbol{n}, \boldsymbol{d} \cdot \boldsymbol{n}+a-b)$ lies outside the non-negative orthant. However, we shall define, for the convenience,

$$
W_{\boldsymbol{d}}(\boldsymbol{n}, a, b)=\left(\begin{array}{c}
a-b+(\boldsymbol{d}+\mathbf{1}) \cdot \boldsymbol{n} \\
\boldsymbol{n}
\end{array}\right)
$$


for $\boldsymbol{n} \geqq \mathbf{0}$ with $0 \leqq \boldsymbol{d} \cdot \boldsymbol{n}<b-a$. Note that the right hand side of the above expression can be called as a negative multinomial coefficient.

In order to derive $W_{\boldsymbol{d}}(\boldsymbol{n}, a, b)$ and its generating function, let us quote the following two lemmas :

Lemma 1. (Mohanty [3]). For any $\beta=\left(\beta_{1}, \cdots, \beta_{k}\right)>\mathbf{0}$, let us put

$$
u(\boldsymbol{X})=u\left(x_{1}, \cdots, x_{k}\right)=\sum_{n \geq 0} A_{n}(1, \boldsymbol{\beta}) \boldsymbol{X}^{n},
$$

where $A_{\boldsymbol{n}}(1, \boldsymbol{\beta})$ is defined by (1.2). Then the function $u(\boldsymbol{X})$ satisfies the equation

$$
u=1+\sum_{i=1}^{k} x_{i} u^{\beta} i
$$

Furthermore the following expansions are valid for any $\alpha$ :

$$
\begin{gathered}
u^{\alpha}=\sum_{n \geq 0} A_{n}(\alpha, \beta) X^{n}, \quad \alpha \neq 0 \\
\frac{u^{\alpha}}{1-\sum_{i=1}^{k} \beta_{i} x_{i} u^{\beta} i^{-1}}=\sum_{n \geq 0}\left(\begin{array}{c}
\alpha+\beta \cdot n \\
n
\end{array}\right) X^{n}
\end{gathered}
$$

Note that the function $u(\boldsymbol{X})$ is the unique solution of (2.2) analytic at $\mathbf{0}$ and that the series appearing in (2.3) and (2.4) converge for $\left|x_{i}\right|<\left|\left(\beta_{i}-1\right)^{\beta_{i}-1} / \beta_{i}^{\beta_{i}}\right|, i=1, \cdots, k$.

LEMMA 2 (Mohanty [3]). For any $\alpha_{1}, \alpha_{2}$ and $\beta>0$, one has the following convolution of Vandermonde's type:

$$
\sum_{0 \leq l \leq n}\left(\begin{array}{c}
\alpha_{1}+\beta \cdot \boldsymbol{l} \\
\boldsymbol{l}
\end{array}\right) A_{n-\boldsymbol{l}}\left(\alpha_{2}, \beta\right)=\left(\begin{array}{c}
\alpha_{1}+\alpha_{2}+\beta \cdot n \\
n
\end{array}\right),
$$

where $\sum_{\mathbf{0} \leq l \leq n}$ means $\sum_{l_{1}=0}^{n_{1}} \cdots \sum_{l_{k}=0}^{n_{k}}$ for $\boldsymbol{l}=\left(l_{1}, \cdots, l_{k}\right)$ and $\boldsymbol{n}=\left(n_{1}, \cdots, n_{k}\right)$.

Since $W_{\boldsymbol{d}}(\boldsymbol{n}, a, \mathbf{0})=A_{\boldsymbol{n}}(\boldsymbol{a}+1, \boldsymbol{d}+\mathbf{1})$ for $\boldsymbol{n} \geqq \mathbf{0}$ according to Mohanty [3], we obtain from Lemma 1

$$
W_{\boldsymbol{d}}(\boldsymbol{X}, a, 0)=\sum_{n \succeq \mathbf{0}} A_{\boldsymbol{n}}(a+1, \boldsymbol{d}+\mathbf{1}) \boldsymbol{X}^{n}=u^{a+1},
$$

where $u(\boldsymbol{X})$ is the unique solution of an equation

$$
u=1+\sum_{i=1}^{k} x_{i} u^{d_{i}+1}
$$

which is analytic at $\mathbf{0 .}$

Note that $W_{\boldsymbol{d}}(\boldsymbol{n}, a, 0)$ is equal to the number of LP's from the origin to $(\boldsymbol{n}, a+\boldsymbol{d} \cdot \boldsymbol{n})$ without crossing a hyperplane $Z_{k+1}=\boldsymbol{d} \cdot \boldsymbol{Z}+a$.

THEOREM 1. For any integers $a, b \geqq 0$ and any $k$-tuple $\boldsymbol{d}>\mathbf{0}$ of integers,

$$
W_{\boldsymbol{d}}(\boldsymbol{n}, a, b)=\sum_{\substack{0 \leq \boldsymbol{l} \leq n \\
\boldsymbol{d} \cdot \boldsymbol{l} \leq b}}\left(\begin{array}{c}
(\boldsymbol{d}+\mathbf{1}) \cdot \boldsymbol{l}-b-1 \\
\boldsymbol{l}
\end{array}\right) A_{n-\boldsymbol{l}}(a+1, \boldsymbol{d}+\mathbf{1}),
$$

where $A_{\boldsymbol{n}}(a+1, \boldsymbol{d}+\mathbf{1})$ is defined by (1.2).

PROOF. For $\boldsymbol{n} \geqq \mathbf{0}$ with $\boldsymbol{d} \cdot \boldsymbol{n}>b$, we get 


$$
W_{\boldsymbol{d}}(\boldsymbol{n}, a, b)=\left(\begin{array}{c}
a-b+(\boldsymbol{d}+\mathbf{1}) \cdot \boldsymbol{n} \\
\boldsymbol{n}
\end{array}\right)-\sum_{\substack{0 \leq \leq \leq \leq n \\
\boldsymbol{d} \cdot \boldsymbol{l} \leq b b}}\left(\begin{array}{c}
(\boldsymbol{d}+\mathbf{1}) \cdot \boldsymbol{l}-b-1 \\
\boldsymbol{l}
\end{array}\right) A_{\boldsymbol{n}-\boldsymbol{l}}(a+1, \boldsymbol{d}+\mathbf{1}),
$$

which yields, from (2.5),

$$
\begin{aligned}
W_{\boldsymbol{d}}(\boldsymbol{n}, a, b)= & \sum_{\boldsymbol{0} \leq \boldsymbol{l} \leq \boldsymbol{n}}\left(\begin{array}{c}
(\boldsymbol{d}+\mathbf{1}) \cdot \boldsymbol{l}-b-1 \\
\boldsymbol{l}
\end{array}\right) A_{\boldsymbol{n}-\boldsymbol{l}}(a+1, \boldsymbol{d}+\mathbf{1}) \\
& -\sum_{\substack{\boldsymbol{0} \leq \boldsymbol{l} \leq n \\
\boldsymbol{d} \cdot \boldsymbol{l}>b}}\left(\begin{array}{c}
(\boldsymbol{d}+\mathbf{1}) \cdot \boldsymbol{l}-b-1 \\
\boldsymbol{l}
\end{array}\right) A_{n-\boldsymbol{l}}(a+1, \boldsymbol{d}+\boldsymbol{l}) \\
= & \sum_{\substack{\boldsymbol{0} \leq \leq \leq n \\
\boldsymbol{d} \cdot \boldsymbol{l} \leq b}}\left(\begin{array}{c}
(\boldsymbol{d}+\mathbf{1}) \cdot \boldsymbol{l}-b-1 \\
\boldsymbol{l}
\end{array}\right) A_{n-\boldsymbol{l}}(a+1, \boldsymbol{d}+\mathbf{1}) .
\end{aligned}
$$

For $\boldsymbol{n} \geqq 0$ with $\boldsymbol{d} \cdot \boldsymbol{n} \leqq b$, it is clear that

$$
W_{\boldsymbol{d}}(\boldsymbol{n}, a, b)=\left(\begin{array}{c}
a-b+(\boldsymbol{d}+\mathbf{1}) \cdot \boldsymbol{n} \\
\boldsymbol{n}
\end{array}\right),
$$

which is equal to the right hand side of (2.8) from (2.5).

THEOREM 2. For any integers $a, b \geqq 0$ and any $k$-tuple $\boldsymbol{d}>\mathbf{0}$ of integers,

$$
W_{\boldsymbol{d}}(\boldsymbol{X}, a, b)=u^{a+1} \varphi_{\boldsymbol{d}}(\boldsymbol{X}, b),
$$

where $\varphi_{\boldsymbol{d}}(\boldsymbol{X}, b)$ is defined by (1.3) and $u^{a+1}$ is given by (2.6).

Proof. From Theorem 1, we get

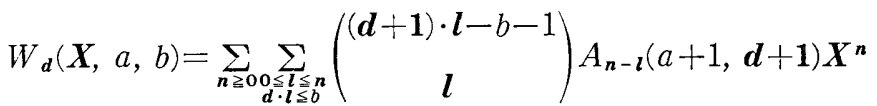

$$
\begin{aligned}
& =\sum_{0 \leq d \cdot l \leq b}\left(\begin{array}{c}
(\boldsymbol{d}+\mathbf{1}) \cdot \boldsymbol{l}-b-1 \\
\boldsymbol{l}
\end{array}\right) \sum_{n \leq l} A_{n-l}(a+1, \boldsymbol{d}+\mathbf{1}) \boldsymbol{X}^{n} \\
& =\sum_{0 \leqq d \cdot l \leqq b}\left(\begin{array}{c}
b-\boldsymbol{d} \cdot \boldsymbol{l} \\
\boldsymbol{l}
\end{array}\right)(-\boldsymbol{X})^{l} \sum_{n \geq 0} A_{n}(a+1, \boldsymbol{d}+\mathbf{1}) \boldsymbol{X}^{n} .
\end{aligned}
$$

From (1.3) and (2.6), we get the equation (2.9).

Corollary 1. For any integers $a, b \geqq 0$ with $b>a$ and any $k$-tuple $\boldsymbol{d}>\mathbf{0}$ of integers,

$$
\sum_{\boldsymbol{d} \cdot \boldsymbol{n}>\boldsymbol{b}-a} W_{\boldsymbol{d}}(\boldsymbol{n}, a, b) \boldsymbol{X}^{\boldsymbol{n}}=u^{a+1} \varphi_{\boldsymbol{d}}(\boldsymbol{X}, b)-\varphi_{\boldsymbol{d}}(\boldsymbol{X}, b-a-1),
$$

where $\varphi_{\boldsymbol{d}}(\boldsymbol{X}, n)$ is defined by (1.3) and $u^{a+1}$ is given by (2.6).

The proof is clear from Theorem 1 and theorem 2 .

\section{Generating Function of $\left\{T_{\boldsymbol{d}}(\boldsymbol{n}, a, b, c), \boldsymbol{n} \geqq \mathbf{0}\right\}$}

In this section, we deal with LP's restricted by two parallel hyperplanes in the $(k+1)$-dimensional lattice space. 
Let us introduce a generating function

$$
T_{\boldsymbol{d}}(\boldsymbol{X}, a, b, c)=\sum_{\boldsymbol{n} \geq \mathbf{0}} T_{\boldsymbol{d}}(\boldsymbol{n}, a, b, c) \boldsymbol{X}^{\boldsymbol{n}},
$$

where $T_{d}(\mathbf{0}, a, b, c)=1$. If $a<b$, we define, for convenience,

$$
T_{\boldsymbol{d}}(\boldsymbol{n}, a, b, c)=W_{\boldsymbol{d}}(\boldsymbol{n}, a, b)=\left(\begin{array}{c}
a-b+(\boldsymbol{d}+\mathbf{1}) \cdot \boldsymbol{n} \\
\boldsymbol{n}
\end{array}\right) \text {, for } \boldsymbol{n} \geqq \mathbf{0} \text { with } 0 \leqq \boldsymbol{d} \cdot \boldsymbol{n}<b-a .
$$

Firstly, we derive $T_{\boldsymbol{d}}(\boldsymbol{X}, b+c, b, c)$ as follows :

THEOREM 3. For any integers $b, c \geqq 0$ and any $k$-tuple $\boldsymbol{d}>\mathbf{0}$ of integers,

$$
T_{\boldsymbol{d}}(\boldsymbol{X}, b+c, b, c)=\frac{\varphi_{\boldsymbol{d}}(\boldsymbol{X}, b)}{\varphi_{\boldsymbol{d}}(\boldsymbol{X}, b+c+1)},
$$

where $\varphi_{\boldsymbol{d}}(\boldsymbol{X}, n)$ is defined by (1.3).

PROOF. For any $\boldsymbol{n} \geqq \mathbf{0}$, we have the following relation :

$$
\sum_{\mathbf{0} \leq l \leq \boldsymbol{n}} T_{\boldsymbol{d}}(\boldsymbol{l}, b+c, b, c) W_{\boldsymbol{d}}(\boldsymbol{n}-\boldsymbol{l}, b+c, b+c+\mathbf{1})=W_{\boldsymbol{d}}(\boldsymbol{n}, b+c, b) .
$$

By applying Theorem 2, we get

$$
T_{\boldsymbol{d}}(\boldsymbol{X}, b+c, b, c)=\frac{u^{b+c+1} \varphi_{\boldsymbol{d}}(\boldsymbol{X}, b)}{u^{b+c+1} \varphi_{\boldsymbol{d}}(\boldsymbol{X}, b+c+1)}=\frac{\varphi_{\boldsymbol{d}}(\boldsymbol{X}, b)}{\varphi_{\boldsymbol{d}}(\boldsymbol{X}, b+c+1)} .
$$

THEOREM 4. For any integers $a, b, c \geqq 0$ with $a \leqq b+c$ and any $k$-tuple $\boldsymbol{d}>\mathbf{0}$ of integers

$$
T_{\boldsymbol{d}}(\boldsymbol{X}, a, b, c)=\frac{\varphi_{\boldsymbol{d}}(\boldsymbol{X}, b) \varphi_{\boldsymbol{d}}(\boldsymbol{X}, b+c-a)}{\varphi_{\boldsymbol{d}}(\boldsymbol{X}, b+c+1)},
$$

where $\varphi_{\boldsymbol{d}}(\boldsymbol{X}, n)$ is defined by (1.3).

Proof. Similarly as in the proof of Theorem 1, it can be seen that

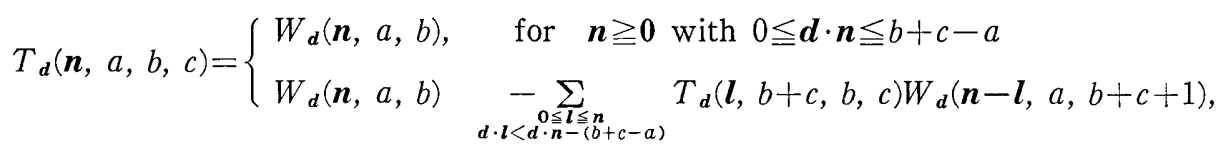

Hence, we obtain

$$
\text { for } \boldsymbol{n} \geqq \mathbf{0} \text { with } \boldsymbol{d} \cdot \boldsymbol{n}>b+c-a .
$$

$$
\begin{aligned}
T_{\boldsymbol{d}}(\boldsymbol{X}, a, b, c) \\
\quad=W_{\boldsymbol{d}}(\boldsymbol{X}, a, b)-\sum_{\boldsymbol{d} \cdot \boldsymbol{n}>b+c-a} \sum_{\substack{\boldsymbol{d} \cdot \boldsymbol{l}<\boldsymbol{d} \cdot \boldsymbol{n}-\boldsymbol{l} \leq \boldsymbol{n}-(b+c-a)\\
}} T_{\boldsymbol{d}}(\boldsymbol{l}, b+c, b, c) W_{\boldsymbol{d}}(\boldsymbol{n}-\boldsymbol{l}, a, b+c+1) \boldsymbol{X}^{\boldsymbol{n}} \\
\quad=W_{\boldsymbol{d}}(\boldsymbol{X}, a, b)-\sum_{\boldsymbol{l} \geq \mathbf{0}} T_{\boldsymbol{d}}(\boldsymbol{l}, b+c, b, c) \sum_{\substack{\boldsymbol{n}: \boldsymbol{l} \\
\boldsymbol{d} \cdot \boldsymbol{n}>\boldsymbol{d} \cdot \boldsymbol{l}+b+c-a}} W_{\boldsymbol{d}}(\boldsymbol{n}-\boldsymbol{l}, a, b+c+1) \boldsymbol{X}^{\boldsymbol{n}} \\
\quad=W_{\boldsymbol{d}}(\boldsymbol{X}, a, b)-T_{\boldsymbol{d}}(\boldsymbol{X}, b+c, b, c) \sum_{\boldsymbol{d} \cdot \boldsymbol{n}>b+c-a} W_{\boldsymbol{d}}(\boldsymbol{n}, a, b+c+1) \boldsymbol{X}^{\boldsymbol{n}} .
\end{aligned}
$$

Appealing to Theorem 2, 3 and Corollary 1, we see that

$$
\begin{aligned}
& T_{\boldsymbol{d}}(\boldsymbol{X}, a, b, c) \\
& \quad=u^{a+1} \varphi_{\boldsymbol{d}}(\boldsymbol{X}, b)-\frac{\varphi_{\boldsymbol{d}}(\boldsymbol{X}, b)}{\varphi_{\boldsymbol{d}}(\boldsymbol{X}, b+c+1)}\left\{u^{a+1} \varphi_{\boldsymbol{d}}(\boldsymbol{X}, b+c+1)-\varphi_{\boldsymbol{d}}(\boldsymbol{X}, b+c-a)\right\}
\end{aligned}
$$




$$
=\frac{\varphi_{\boldsymbol{d}}(\boldsymbol{X}, b) \varphi_{\boldsymbol{d}}(\boldsymbol{X}, b+c-a)}{\varphi_{\boldsymbol{d}}(\boldsymbol{X}, b+c+1)} .
$$

Now we show that $T_{\boldsymbol{d}}(\boldsymbol{X}, a, b, c)$ converges to $W_{\boldsymbol{d}}(\boldsymbol{X}, a, b)$ as $c \rightarrow \infty$. To this end, we prove the following lemma.

LEMMA 3. For any $k$-tuple $\boldsymbol{d}>\mathbf{0}$ of integers,

$$
\lim _{n \rightarrow \infty} \varphi_{\boldsymbol{d}}(\boldsymbol{X}, n) u^{n+1}=\frac{1}{1-\sum_{i=1}^{k}\left(d_{i}+1\right) x_{i} u^{d_{i}}},
$$

where $u(\boldsymbol{X})$ is given by (2.7) and $\left|x_{i}\right|<d_{i}^{d} i /\left(d_{i}+1\right)^{\left(d_{i}+1\right)}, i=1,2, \cdots, k$, for $\boldsymbol{X}=\left(x_{1}, \cdots, x_{k}\right)$.

Proof. From (2.4), we get

$$
\frac{1}{1-\sum_{i=1}^{k}\left(d_{i}+1\right) x_{i} u^{d_{i}}}=\sum_{l \geq 0}\left(\begin{array}{c}
(\boldsymbol{d}+\mathbf{1}) \cdot \boldsymbol{l} \\
\boldsymbol{l}
\end{array}\right) \boldsymbol{X}^{l},
$$

which can be rewritten as

$$
\begin{aligned}
& \frac{1}{1-\sum_{i=1}^{k}\left(d_{i}+1\right) x_{i} u^{d_{i}}}=\frac{u^{-(n+1)}}{1-\sum_{i=1}^{k}\left(d_{i}+1\right) x_{i} u^{d_{i}}} u^{n+1} \\
& =\sum_{\boldsymbol{l} \leq \mathbf{0}}\left(\begin{array}{c}
(\boldsymbol{d}+\mathbf{1}) \cdot \boldsymbol{l}-n-1 \\
\boldsymbol{l}
\end{array}\right) \boldsymbol{X}^{\boldsymbol{l}} u^{n+1} \\
& =\sum_{0 \leq \boldsymbol{d} \cdot \boldsymbol{l} \leq n}\left(\begin{array}{c}
(\boldsymbol{d}+\mathbf{1}) \cdot \boldsymbol{l}-n-1 \\
\boldsymbol{l}
\end{array}\right) \boldsymbol{X}^{\boldsymbol{l}} u^{n+1}+\sum_{\boldsymbol{d} \cdot \boldsymbol{l}>n}\left(\begin{array}{c}
(\boldsymbol{d}+\mathbf{1}) \cdot \boldsymbol{l}-n-1 \\
\boldsymbol{l}
\end{array}\right) \boldsymbol{X}^{\boldsymbol{l}} u^{n+1} \\
& =\varphi_{\boldsymbol{d}}(\boldsymbol{X}, n) u^{n+1}+\sum_{\boldsymbol{d} \cdot \boldsymbol{l}>n}\left\{\sum_{\substack{\boldsymbol{d}, \boldsymbol{m}>n \\
\boldsymbol{0} \leq \boldsymbol{m} \leq \boldsymbol{l}}}\left(\begin{array}{c}
(\boldsymbol{d}+\boldsymbol{l}) \cdot \boldsymbol{m}-n-1 \\
\boldsymbol{m}
\end{array}\right) A_{\left.\boldsymbol{l}-\boldsymbol{m}(n+1, \boldsymbol{d}+\mathbf{1}) \boldsymbol{X}^{\boldsymbol{l}}\right\}}\right.
\end{aligned}
$$

for any $n \geqq 0$. The coefficient of $\boldsymbol{X}^{\boldsymbol{l}}$ in the expansion of $\varphi_{\boldsymbol{d}}(\boldsymbol{X}, n) u^{n+1}$ is positive for any $n \geqq 0$ since it is given by $W_{\boldsymbol{d}}(\boldsymbol{l}, n, n)$. It follows that

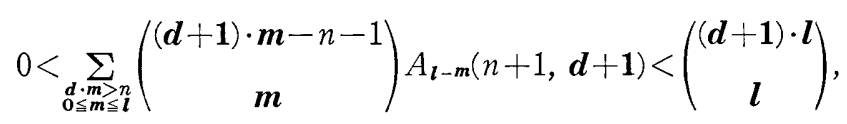

for any $n \geqq 0$ and any $\boldsymbol{l} \geqq \mathbf{0}$ with $\boldsymbol{d} \cdot \boldsymbol{l}>n$. Since $\lim _{n \rightarrow \infty} \sum_{\boldsymbol{d} \cdot \boldsymbol{l}>n}\left(\begin{array}{c}(\boldsymbol{d}+\mathbf{1}) \cdot \boldsymbol{l} \\ \boldsymbol{l}\end{array}\right) \boldsymbol{X}^{\boldsymbol{l}}=0$ for $\left|x_{i}\right|<$ $d_{i}^{d} i /\left(d_{i}+1\right)^{d_{i+1}}, i=1, \cdots, k$, the second term of the right right hand side of (3.6) converges to 0 . Thus,

$$
\lim _{n \rightarrow \infty} \varphi_{\boldsymbol{d}}(\boldsymbol{X}, n) u^{n+1}=\frac{1}{1-\sum_{i=1}^{k}\left(d_{i}+1\right) x_{i} u^{d_{i}}} .
$$

From Lemma 3, it follows immediately that:

THEOREM 5. For any integers $a, b \geqq 0$ any $k$-tuple $\boldsymbol{d}>\mathbf{0}$ of integers,

$$
\lim _{c \rightarrow \infty} T_{\boldsymbol{d}}(\boldsymbol{X}, a, b, c)=W_{\boldsymbol{d}}(\boldsymbol{X}, a, b),
$$

for $\left|x_{i}\right|<d_{i}^{d} i_{(} /\left(d_{i}+1\right)^{\left(d_{i}+1\right)}, i=1,2, \cdots, k$. 


\section{Explicit Expressions}

In this section, we shall give explicit expressions of both $W_{\boldsymbol{d}}(\boldsymbol{n}, a, b)$ and $T_{\boldsymbol{d}}(\boldsymbol{n}, a, b, c)$ for a special case that $\boldsymbol{d}=\mathbf{1}$.

For $\boldsymbol{d}=\mathbf{1}$, if one puts $Z=\sum_{i=1}^{k} Z_{i}$ and $n=\sum_{i=1}^{k} n_{i}$ for $\boldsymbol{n}=\left(n_{1}, \cdots, n_{k}\right)$ he may easily see that

$$
\begin{aligned}
& W_{1}(\boldsymbol{n}, a, b)=\left(\begin{array}{l}
n \\
\boldsymbol{n}
\end{array}\right) W_{1}(n, a, b) \\
& T_{\mathbf{1}}(\boldsymbol{n}, a, b, c)=\left(\begin{array}{l}
n \\
\boldsymbol{n}
\end{array}\right) T_{1}(n, a, b, c),
\end{aligned}
$$

where, as defined before, $W_{1}(n, a, b)$ is the number of LP's from the origin to the point $(n, n+a-b)$ without crossing a line $Z_{k+1}=Z-b$ in the 2-dimensional space $\left(Z, Z_{k+1}\right)$ and $T_{1}(n, a, b, c)$ that of LP's restricted by two lines $Z_{k+1}=Z-b$ and $Z_{k+1}=z+c$. Therefore we have

$$
\begin{aligned}
& W_{1}(\boldsymbol{X}, a, b)=\sum_{n=0}^{\infty} W_{1}(n, a, b)\left(\sum_{i=1}^{k} x_{i}\right)^{n} \\
& T_{1}(\boldsymbol{X}, a, b)=\sum_{n=0}^{\infty} T_{1}(n, a, b, c)\left(\sum_{i=1}^{k} x_{i}\right)^{n} .
\end{aligned}
$$

In the present section, we will drive $W_{1}(\boldsymbol{n}, a, b)$ and $T_{1}(\boldsymbol{n}, a, b, c)$ using the generating functions obtained in the previous sections, respectively.

THEOREM 6. For any integers $a, b \geqq 0$,

(i) $W_{1}(\boldsymbol{X}, a, b)=\left(\frac{1-\sqrt{1-4 x}}{2 x}\right)^{a+1} x^{b / 2} U_{b}\left(\frac{1}{2 \sqrt{x}}\right)$

(ii) $W_{\mathbf{1}}(\boldsymbol{n}, a, b)=\left\{\left(\begin{array}{c}2 n+a-b \\ n\end{array}\right)-\left(\begin{array}{c}2 n+a-b \\ n-b-1\end{array}\right)\right\}\left(\begin{array}{l}n \\ \boldsymbol{n}\end{array}\right), \quad \boldsymbol{n} \geqq \mathbf{0}$,

where $x=\sum_{i=1}^{k} x_{i}$ and $n=\sum_{i=1}^{k} n_{i}$ for $\boldsymbol{X}=\left(x_{1}, \cdots, x_{k}\right)$ and $\boldsymbol{n}=\left(n_{1}, \cdots, n_{k}\right)$, respectively, and $U_{n}(x)$ is the Chebyshev polynomial of the second kind.

Proof. From Theorem 2, we get

$$
W_{1}(\boldsymbol{X}, a, b)=\varphi_{1}(\boldsymbol{X}, b) u^{a+1},
$$

where $u(\boldsymbol{X})$ is the unique solution with $u(\mathbf{0})=1$ of

$$
u=1+\sum_{i=1}^{k} x_{i} u^{2}
$$

and $\varphi_{1}(\boldsymbol{X}, b)$ is given by

$$
\varphi_{1}(X, b)=\sum_{0 \leq 1 \cdot l \leq b}\left(\begin{array}{c}
b-1 \cdot l \\
l
\end{array}\right)(-X)^{l} .
$$

(4.1) is valid for any $a, b \geqq 0$ since it follows that 


$$
u^{a+1}=\left(\frac{1-\sqrt{1-4 x}}{2 x}\right)^{a+1}=\sum_{n=0}^{\infty} A_{n}(a+1,2) x^{n}
$$

and

$$
\varphi_{1}(\boldsymbol{X}, b)=\sum_{l=0}^{[b / 2]}\left(\begin{array}{c}
b-l \\
l
\end{array}\right)(-x)^{l}=x^{b / 2} U_{b}\left(\frac{1}{2 \sqrt{x}}\right),
$$

where $x=\sum_{i=1}^{k} x_{i}, A_{n}(a+1,2)=\frac{a+1}{a+1+2 n}\left(\begin{array}{c}a+1+2 n \\ n\end{array}\right)$ for $n \geqq 0$ and $U_{b}$ is the Chebyshev polynomial of the second kind:

$$
U_{b}(\cos \theta)=\frac{\sin (b+1) \theta}{\sin \theta}
$$

From (4.3) and (4.4), we have

$$
\begin{aligned}
W_{\mathbf{1}}(\boldsymbol{X}, a, b) & =\sum_{n=0}^{\infty} \sum_{l=0}^{\min [n, b]}(-1)^{l}\left(\begin{array}{c}
b-l \\
l
\end{array}\right) A_{n-l}(a+1,2) x^{n} \\
& =\sum_{n=0}^{\infty}\left\{\left(\begin{array}{c}
2 n+a-b \\
n
\end{array}\right)-\left(\begin{array}{c}
2 n+a-b \\
n-b-1
\end{array}\right)\right\} x^{n},
\end{aligned}
$$

which implies, with $x=\sum_{i=1}^{k} x_{i}$,

$$
W_{1}(\boldsymbol{n}, a, b)=\left\{\left(\begin{array}{c}
2 n+a-b \\
n
\end{array}\right)-\left(\begin{array}{c}
2 n+a-b \\
n-b-1
\end{array}\right)\right\}\left(\begin{array}{l}
n \\
\boldsymbol{n}
\end{array}\right), \quad \boldsymbol{n} \geqq \mathbf{0}
$$

with the convention $\left(\begin{array}{l}n \\ r\end{array}\right)=0$ for $r<0$. Similarly, it follows that

THEOREM 7. For any integers $a, b, c \geqq 0$ with $0 \leqq a \leqq b+c$,

(i) $T_{\mathbf{1}}(\boldsymbol{X}, a, b, c)=x^{(b-a-1) / 2} \frac{U_{b}\left(\frac{1}{2 \sqrt{x}}\right) U_{b+c-a}\left(\frac{1}{2 \sqrt{x}}\right)}{U_{b+c+1}\left(\frac{1}{2 \sqrt{x}}\right)}$

(ii) $T_{1}(\boldsymbol{n}, a, b, c)=\frac{4}{b+c+2}\left\{\sum_{\nu=1}^{M}\left(2 \cos \theta_{\nu}\right)^{2 n+a-b} \sin (b+1) \theta_{\nu} \sin (a+1) \theta_{\nu}\right\}\left(\begin{array}{l}n \\ \boldsymbol{n}\end{array}\right)$,

$$
n \geqq b-a,
$$

where $U_{n}$ is the Chebyshev polynomial of the second kind, $x=\sum_{i=1}^{k} x_{i}, n=\sum_{i=1}^{k} n_{i}$, and $\theta_{\nu}=$ $\frac{\nu \pi}{b+c+2}$ for $\nu=1,2, \cdots,[(b+c+1) / 2](=M)$.

Proof. It is clear that (4.5) is immediately follows from Theorem 3 and (4.4). Putting $x=(2 \cos \theta)^{-2}, 0<\theta<\pi / 2$, we get

$$
T_{1}(X, a, b, c)=(2 \cos \theta)^{-(b-a-1)} \frac{\sin (b+1) \theta \sin (b+c-a+1) \theta}{\sin \theta \sin (b+c+2) \theta} .
$$

There are $[(b+c+1) / 2]$ roots of $\varphi_{1}(\boldsymbol{X}, b+c+1)$ distinct from each other :

$$
x^{(2)}=\left(2 \cos \theta_{\nu}\right)^{-2} \text {, }
$$


where $\theta_{\nu}=\frac{\nu \pi}{b+c+2}, \nu=1, \cdots,[(b+c+1) / 2](=M)$. Consequently, the coefficient of $x^{n}$ in the expansion of $T_{1}(\boldsymbol{X}, a, b, c)$ is given by

$$
\frac{4}{b+c+2} \sum_{\nu=1}^{M}\left(2 \cos \theta_{\nu}\right)^{2 n+a-b} \sin (b+1) \theta_{\nu} \sin (a+1) \theta_{\nu}
$$

for $n \geqq b-a$. From $x^{n}=\sum_{1 \cdot n=n}\left(\begin{array}{l}n \\ n\end{array}\right) X^{n}$, we obtain (4.6).

\section{Acknowledgement}

We wish to express our thanks to Professor T. Ishihara for his valuable discussion and encouragement.

\section{References}

1. S.G. MonAwry: Lattice path counting and applications, Probability and Mathematical Statistics (Z.W. Birnbaum and E. Lukacs, ed.). Academic Press, N. Y., (1979).

2 S.G. Moliaxty: An urn problem related to the ballot problem. Amer. Math. Monthly, 73 (1966), 526-528.

3 . S.G. Mohanty: Some convolutions with multinomial coefficients and related probability. SIAM Review, 8 (1966), 501-509.

4- S.G. Mohanty: On queues involving batches. Jour. Applied Probability, 9 (1972), 430-435.

5 H.W. Golld: Some generalizations of Vandermonde's convoltion. Amer. Math. Month., 63 (1956), 84-91.

6. H.W. Golld: Final analysis of Vandermonde's convolution. Amer. Math. Month., 64 (1957), 409-415.

7 H.W. Golld: Coefficient identities for powers of Taylor and Dirichlet series. Amer. Math. Month., 81 (1974), 3-13.

8. H.W. Golld and J. KAUCKY: Evaluation of a class of binomial coefficient summations. Jour. Combinatorial Theory, 1 (1966), 233-247.

9. D.G. Rogers: Pascal triangles, Catalan numbers and renewal arrays. Discrete Math., 22 (1978), 301-310.

10. J.H. VAN LINT: Combinatorial theory seminar. Lec. Notes in Math., Springer-Verlag, 382 (1974).

11. D.E. KNCTH: Fundamental algorithms. Addition-Wesley, 2nd edition, (1973).

12] I. SENBA: On the number of permutations obtainable from a stack restricted by length and its generating function. Sugaku, (in Japanese), Vol. 33, No. 1 (1981), 79-80.

13. D. Singmaster: An elementary evolution of the Catalan numbers. Amer. Math. Month., 85 (1978), 366-368.

[14. T.P. SPEED: Geometric and probabilistic aspects of some combinatorial identities. Jour. Austral. Math. Soc., 22 (1976), 462-468.

15. T.T. CoNG and M. SATO: One-dimensional random walk with unequal step length restricted by an absorbing barrier. Discrete Math., 40 (1982), 153-162.

16. M. SATO and T.T. CONG: The number of minimal lattice paths restricted by two parallel lines. Discrete Math., 43 (1983), 249-261.

Communicated by T. Kitagawa

Received October 1, 1984 